\title{
ANTICANCER DRUGS
}

\section{Targeting menin}

Targeting MLLdriven leukaemia might be possible using drugs that target the MLLmenin interactio.
The mixed lineage leukaemia $(M L L)$ gene, which encodes a histone methyltransferase, is frequently translocated in human acute leukaemias. The interaction of MLL with menin is known to be essential for the oncogenic activity of MLL-fusion proteins; therefore, targeting this interaction could have therapeutic relevance.

Jolanta Grembecka, Tomasz Cierpicki and colleagues have previously characterized the interaction between MLL and menin, which requires the amino terminus of

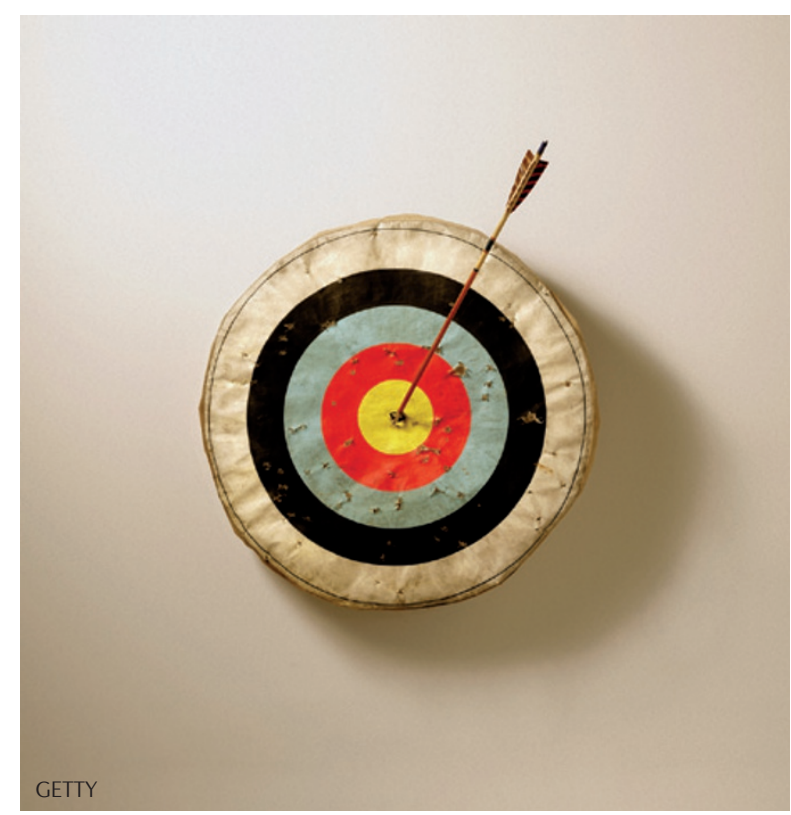

MLL, and this part of the protein remains intact in all MLL-fusion proteins studied to date. The authors used high-throughput screening to identify compounds that target menin and that suppress its interaction with MLL. The most potent compound, a thienopyrimidine dubbed MI-1, binds menin and is a competitive inhibitor of the MLL-menin interaction. Structureactivity analyses led to the generation of MI-1 analogues, MI-2 and MI-3. These molecules bind to wild-type menin - but not to menin mutants in which the interaction site with MLL is mutated - and were taken forwards for further testing.

Co-immunoprecipitation experiments showed that, compared with a control compound, both MI-2 and MI-3 inhibited the interaction between leukaemic MLL-fusion proteins and menin, and this inhibition promoted the differentiation of leukaemia cells and reduced their ability to form colonies in soft agar. MLL-fusion proteins are thought to promote leukaemogenesis partly by enabling the transcription of HOX genes. The treatment of mouse bone marrow cells that expressed an MLLfusion protein with either MI-2 or MI-3 downregulated the expression of Hoxa9 and the HOX cofactor Meis1. Further experiments showed that MI-2 reduced the levels of menin and an MLL-AF9-fusion protein bound at the Hoxa9 promoter, and this was associated with chromatin condensation. Similar results, including reduced proliferation and increased cell differentiation and apoptosis, were evident in human leukaemia cells with MLL-fusion genes. Interestingly, MI-2 and MI-3 also reduced proliferation in leukaemia cells that had high levels of HOX gene expression in the absence of MLL-fusion proteins. The authors speculate that this might be because the interaction between wild-type MLL and menin is prevented on treatment of these cells, and that this interaction is required for HOX gene transcription.

These results indicate that targeting MLL-driven leukaemia might be possible using drugs that target the MLL-menin interaction. MI-2 and MI-3 should prove useful agents for the development of clinically useful inhibitors and for gaining a better understanding of the function of the MLL-menin interaction in leukaemogenesis.

Nicola McCarthy Chief Editor, Nature Reviews Cancer This article originally appeared in Nature Rev. Cancer (doi:10.1038/nrc3231).

ORIGINAL RESEARCH PAPER Grembecka, J. et al. Menin-MLL inhibitors reverse oncogenic activity of MLL fusion proteins in leukaemia. Nature Chem. Biol. 29 Jan 2012 (doi:10.1038/nchembio.773) 